\title{
Improving Convergence Rate in the Method of Successive Approximations*
}

\author{
By James A. Pennline
}

\begin{abstract}
An application of the method of successive approximations for obtaining the solution of a nonlinear integral equation arising from a two-point boundary value problem is illustrated. In particular, we show sufficient conditions under which the convergence rate of the sequence can be improved.
\end{abstract}

1. Introduction. Consider two-point boundary value problems of the form

$$
\begin{gathered}
u^{\prime \prime}=f(x, u), \quad 0<x<1, \\
u(0)=u(1)=0 .
\end{gathered}
$$

One method for solving such problems is to convert (1) to an integral equation which under sufficient conditions can be solved by successive approximations. This method is discussed by Keller [3]. His approach is to first subtract $k^{2} u$ from both sides of (1a) and consider the equivalent problem

$$
u^{\prime \prime}-k^{2} u=f(x, u)-k^{2} u, \quad u(0)=u(1)=0 .
$$

Then, for $k \neq 0$, (2) can be converted to an integral equation by the Green's function procedure for the operator $\left(d^{2} / d x^{2}-k^{2}\right)$, i.e.,

$$
u(x)=\int_{0}^{1} g_{k}(x, \xi)\left\{k^{2} u(\xi)-f(\xi, u(\xi))\right\} d \xi
$$

where

$$
g_{k}(x, \xi)=\frac{1}{k \sinh k} \begin{cases}\sinh k x \sinh k(1-\xi), & 0 \leqslant x<\xi \\ \sinh k(1-x) \sinh k \xi, & \xi<x<1 .\end{cases}
$$

The advantage gained in considering the equivalent problem (2) in place of (1) is seen in the following result given by Keller [3].

TheOREM (Keller). Let $\partial f / \partial u$ be continuous for all $x \in[0,1]$ and all $u$. If there exists an $N>0$ such that $0 \leqslant \partial f(x, u) / \partial u<N$ for all $x \in[0,1]$ and all $u$, then there exists a unique solution of (1). For any $k$ such that $k^{2}>N$, the solution is given by the limit of the convergent sequence of functions

$$
u^{0}(x)=0 \text {, }
$$

$$
u^{m+1}(x)=\int_{0}^{1} g_{k}(x, \xi)\left[k^{2} u^{m}(\xi)-f\left(\xi, u^{m}(\xi)\right)\right] d \xi, \quad m=0,1,2, \ldots
$$

Received July 10, 1979; revised December 5, 1979 and November 11, 1980.

AMS (MOS) subject classifications (1970). Primary 65R05, 45G99, 65L10, 34B 15.

Key words and phrases. Boundary value problem, integral equation, successive approximations, convergence rate.

- This work was done in part while the author was on a summer faculty fellowship, Computer Science Division, NASA Lewis Research Center, Cleveland, Ohio. 
Proof. Let

$$
e^{m+1}(x)=u^{m+1}(x)-u^{m}(x)
$$

and

$$
\left\|e^{m+1}\right\|=\max _{0<x<1}\left|e^{m+1}(x)\right|, \quad m=0,1, \ldots
$$

Then, for $m=1,2, \ldots$,

$$
e^{m+1}(x)=\int_{0}^{1} g_{k}(x, \xi)\left[k^{2}-\frac{\partial f}{\partial u}\left(\xi, u^{m}(\xi)-\theta(\xi) e^{m}(\xi)\right)\right] e^{m}(\xi) d \xi,
$$

where the mean-value theorem has been applied and $0<\theta(\xi)<1$. Note that $g_{k}(x, \xi) \geqslant 0$, and $0 \leqslant \partial f / \partial u \leqslant N$ is assumed for all $u$. Therefore, if $k^{2}>N$, the bracketed term in the integrand of (7) satisfies $0<\left(k^{2}-\partial f / \partial u\right)<k^{2}$. Hence,

$$
\begin{aligned}
\left|e^{m+1}(x)\right| & \leqslant k^{2} \int_{0}^{1} g_{k}(x, \xi) d \xi \cdot\left\|e^{m}\right\| \\
& \leqslant \mu_{k}\left\|e^{m}\right\|, \quad m=1,2, \ldots,
\end{aligned}
$$

where

$$
\mu_{k}=\left(1-\frac{1}{\cosh (k / 2)}\right)
$$

Since Eq. (8) holds for all $x$, we have

$$
\left\|e^{m+1}\right\| \leqslant \mu_{k}\left\|e^{m}\right\|, \quad m=1,2, \ldots
$$

Observe that $\mu_{k}<1$, and $\left\|e^{m+1}\right\| \leqslant \mu_{k}^{m}\left\|e^{1}\right\|$. Thus $\left\{u^{m}\right\}$ is a Cauchy sequence in the space of continuous functions on $[0,1]$ with the norm defined by (6). Therefore, a continuous limit $u$ exists, to which $\left\{u^{m}(x)\right\}$ converges uniformly. Since the order of the limit operation and the integration can be interchanged, the limit function satisfies the integral equation (3). To establish uniqueness, let $u(x)$ and $v(x)$ be two solutions to (1). Then they both satisfy (3) for the same value of $k^{2}>N$. By the same analysis that leads to (10), $\|u-v\| \leqslant \mu_{k}\|u-v\|$. Since $\mu_{k}<1$, we must have $\|u-v\|=0$ or $u=v$.

The existence portion of Keller's proof is constructive in nature and suggests a procedure for computing the solution numerically. However, as Keller points out, $\mu_{k}$ may be very close to one for all values of $k \geqslant N$. This could make the rate of convergence of the sequence (4) very slow. In practice, such cases can cause the number of iterations required for a given error tolerance, when programming the successive approximations, to be large. Keller [3] suggests Newton's method as an alternative scheme when $\mu_{k}$ is nearly one. The purpose of this paper is to illustrate conditions under which the parameter $\mu_{k}$ in (9) can be replaced by a much smaller parameter when the above method is applied to suitable two-point boundary value problems. A recent application is also given.

2. Improved Convergence Rate. We first make the observation that Keller's proof and conclusions are unaltered if his condition $k^{2}>N$ is replaced by the weaker condition $k^{2} \geqslant \frac{1}{2} N$. This is because, in the proof, the bracketed term in the integral (7) can then become negative, but will satisfy $0 \leqslant\left|k^{2}-\partial f / \partial u\right|<k^{2}$. Thus the step leading from (7) to (8) will remain the same. However, with this change in the condition on $k^{2}, \mu_{k}$ can be made smaller than any value of $\mu_{k}$ allowed by $k^{2}>N$. 
This can be done for instance by choosing

$$
k^{2}=\frac{1}{2} N
$$

As a matter of fact, this choice gives the smallest value of $\mu_{k}$ for all $k^{2}>\frac{1}{2} N$.

Next, we illustrate a set of conditions which includes the class of functions $f(x, u)$, described by Keller's assumption, and which can also distinguish between slightly more restrictive subsets of that class. We shall show that one particular choice of $k$, which reduces to (11) when the conditions admit the class of functions $f$ in Keller's theorem, will always give a contractive parameter smaller than $\mu_{k}$ with $k>N$.

THEOREM 1. In the boundary value problem (1), let $\partial f / \partial u$ be continuous for all $x \in[0,1]$ and all $u$. Suppose that there exists $N>0$ and $\delta>0$ such that $0<\delta<$ $\partial f / \partial u \leqslant N$ for all $x \in[0,1]$ and all $u$. Then a unique solution of (1) exists. For $k^{2}=\frac{1}{2}(\delta+N)$, it is given by the limit of the convergent sequence (4).**

Proof. In terms of definitions (5) and (6), formula (7) again follows. With the choice

$$
k^{2}=\frac{1}{2}(\delta+N)
$$

and the bounds on $\partial f / \partial u$, we have $0 \leqslant\left|k^{2}-\partial f / \partial u\right|<\frac{1}{2}(N-\delta)$. Therefore, from (7),

$$
\begin{aligned}
\left|e^{m+1}(x)\right| & \leqslant \int_{0}^{1} g_{k}(x, \xi) \frac{1}{2}(N-\delta) d \xi\left\|e^{m}\right\| \\
& =\frac{1}{2}(N-\delta) \cdot \frac{1}{k^{2}}\left(1-\frac{\cosh k\left(\frac{1}{2}-x\right)}{\cosh (k / 2)}\right) \cdot\left\|e^{m}\right\| \\
& \leqslant \frac{N-\delta}{N+\delta} \cdot\left(1-\frac{1}{\cosh (k / 2)}\right) \cdot\left\|e^{m}\right\|, \quad m=1,2, \ldots
\end{aligned}
$$

Since this relation holds for all $x \in[0,1]$,

$$
\left\|e^{m+1}\right\| \leqslant \bar{\mu}_{k}\left\|e^{m}\right\|, \quad m=1,2, \ldots,
$$

where

$$
\bar{\mu}_{k}=\frac{N-\delta}{N+\delta}\left(1-\frac{1}{\cosh (k / 2)}\right) .
$$

Observe that $\bar{\mu}_{k}<1$, so that, by the same arguments as those in Keller's theorem, the sequence $\left\{u^{m}(x)\right\}$ has a limit which is the unique solution of (1).

Compare the contractive parameter $\bar{\mu}_{k}$ of (15) to the contractive parameter $\mu_{k}$ of (9). Note that, in the case $0=\delta$, the condition on $\partial f / \partial u$ is the same as the condition in Keller's theorem, but $\bar{\mu}_{k}$ will be defined with $k^{2}=\frac{1}{2} N$ and will be smaller than any value of $\mu_{k}$ defined by $k^{2}>N$. If $0<\delta<N$, then the class of functions $f$ admitted by Theorem 1 is a particular subset of the class defined in Keller's theorem. Also, $\bar{\mu}_{k}$ is defined with $k^{2}=\frac{1}{2}(\delta+N)$ and is again smaller than any value of $\mu_{k}$ with $k^{2} \geqslant N$. In some cases, $\bar{\mu}_{k}$ may be significantly smaller, and, in practice, the iterative procedure used on the sequence $\left\{u^{m}(x)\right\}$ will converge much faster. To see this, some specific cases will be shown in Section 3.

** The condition $k^{2}=\frac{1}{2}(\delta+N)$ can be replaced by the weaker condition $k^{2}>\frac{1}{2}(\delta+N)$. However, the purpose above is to emphasize an optimal choice for convergence speed. 
Before we illustrate an example application of the above result, an important weakening of the assumptions needs to be discussed. Both Keller's theorem and Theorem 1 require that $f$ satisfy appropriate conditions for all $u$. In many cases, such as the application in Section 3, $f$ may not satisfy these conditions for all $u$. However, the method can still be applied if certain additional conditions are satisfied. For a simple example, consider the following.

Theorem 2. Let $M>0$ and $N>0$. Suppose that $\partial f / \partial u$ is continuous and satisfies $0 \leqslant \delta \leqslant \partial f / \partial u \leqslant N$ for all $x \in[0,1]$ and all $u$ such that $0<u(x)<M, 0<x<1$. Suppose further that $0 \leqslant f(x, u) \leqslant \frac{1}{2}(\delta+N) \cdot u$ for all $x \in[0,1]$ and all $u$ such that $0 \leqslant u(x)<M, 0 \leqslant x \leqslant 1$. Then there exists a unique solution of (1) satisfying $0<u(x)<M, x \in[0,1]$. For $k^{2}=\frac{1}{2}(\delta+N)$ it is given by the limit of the convergent sequence of functions (4).

Proof. It is sufficient to show that each member of the sequence (4) satisfies $0<u^{m}(x) \leqslant M, x \in[0,1]$. Then the proof of Theorem 1 is applicable. To this end observe that $u^{0} \equiv 0$ and satisfies $0 \leqslant u^{0}<M$. Now assume that $u^{r}(x)$ satisfies $0 \leqslant u^{r}(x) \leqslant M, 0 \leqslant x \leqslant 1$, for some $r \geqslant 0$. Since $f\left(x, u^{r}\right)<\frac{1}{2}(N+\delta) u^{r}, 0<k^{2} u^{r}$ $-f\left(x, u^{r}\right)$ if $k^{2} \geqslant \frac{1}{2}(N+\delta)$. Also, $g_{k}(x, \xi)$ is nonnegative on $[0,1]$. Thus the integrand in (4b) is nonnegative and $0<u^{r+1}(x), 0<x<1$. Since $u^{r}(x)<M$, $f\left(x, u^{r}\right)<\frac{1}{2}(N+\delta) M$. If $k^{2}=\frac{1}{2}(N+\delta)$, then, from $(4 \mathrm{~b})$,

$$
\begin{aligned}
u^{r+1}(x) & \leqslant \int_{0}^{1} k^{2} M g_{k}(x, \xi) d \xi \\
& =k^{2} M\left(1-\frac{\cosh k\left(\frac{1}{2}-x\right)}{\cosh k / 2}\right) \frac{1}{k^{2}}<M
\end{aligned}
$$

for all $x \in[0,1]$. Thus, by induction, $0 \leqslant u^{m}(x)<M, m=0,1, \ldots$

As an additional remark, we point out that results similar to the above can be stated for two-point problems with Neumann end conditions $u^{\prime}(0)=u^{\prime}(1)=0$ or mixed end conditions $u^{\prime}(0)=u(1)=0$ as well as for cases in which the boundary conditions are nonhomogeneous.

3. Application. The problem of steady-state, isothermal, reaction-diffusion of a substance involving $n$th order kinetics [1] leads to the two-point problem

$$
\begin{gathered}
u^{\prime \prime}=\phi^{2} u^{n}, \quad n \geqslant 1, \quad \phi^{2} \neq 0, \\
u^{\prime}(0)=0, \quad u(1)=1,
\end{gathered}
$$

in dimensionless variables. Here $\phi^{2}$ is a nonzero constant dependent on geometry, concentration, and reaction rate. In [1], an asymptotic analysis is applied to (16). The problem is also studied in [2] where a limited approximation is developed as well as an iterative numerical procedure which uses the ideas suggested in Section 2.

We first make note that the case $n=1$ can be solved by elementary methods to yield

$$
u(x)=\frac{\cosh \phi x}{\cosh \phi}, \quad n=1 \text {. }
$$

We will therefore consider cases $n \geqslant 2$. Also, on physical grounds, solutions for $n>1$ are expected to satisfy $\cosh \phi x / \cosh \phi<u(x)<1$. Solutions which satisfy this condition will be sought. 
Following the approach outlined in the introduction, we consider

$$
\begin{gathered}
u^{\prime \prime}-k^{2} u=\phi^{2} u^{n}-k^{2} u, \\
u^{\prime}(0)=0, \quad u(1)=1 .
\end{gathered}
$$

Then for $k \neq 0,(18)$ can be converted by the Green's function method to

$$
u(x)=\frac{\cosh k x}{\cosh k}+\int_{0}^{1} g_{k}(x, \xi)\left[k^{2} u(\xi)-\phi^{2}[u(\xi)]^{n}\right] d \xi
$$

where

$$
g_{k}(x, \xi)=\frac{1}{k \cosh k} \begin{cases}\sinh k(1-\xi) \cosh k x, & 0<x<\xi, \\ \cosh k \xi \sinh k(1-x), & \xi<x<1 .\end{cases}
$$

For application of the ideas in Section 2, we form the sequence

$$
\begin{gathered}
u^{0}(x)=\frac{\cosh \phi x}{\cosh \phi}, \\
u^{m+1}(x)=\frac{\cosh k x}{\cosh k}+\int_{0}^{1} g_{k}(x, \xi)\left[k^{2} u^{m}(\xi)-\phi^{2}\left[u^{m}(\xi)\right]^{n}\right] d \xi, \\
m+0,1, \ldots * * *
\end{gathered}
$$

Now, in order to show how conditions similar to the conditions in Theorem 2 can be satisfied, we demonstrate the following.

(a) For this problem, $f(x, u)=\phi^{2} u^{n}$ and

$$
0<n \phi^{2}\left(\frac{1}{\cosh \phi}\right)^{n-1} \leqslant \partial f(x, u) / \partial u<n \phi^{2},
$$

for all $x \in[0,1]$ and all $u$ such that $1 / \cosh \phi \leqslant u(x)<1$ in $[0,1]$. Note that $n \phi^{2}(1 / \cosh \phi)^{n-1}$ plays the role of $\delta>0$, and $n \phi^{2}$ plays the role of $N$.

(b) For all $x \in[0,1]$ and all $u$ such that $1 / \cosh \phi<u(x)<1$ in $[0,1]$,

$$
0<\phi^{2}(1 / \cosh \phi)^{n}<f(x, u)<\phi^{2} .
$$

(c) Note the upper bound $N$ and the lower bound $\delta$ for $\partial f / \partial u$ indicated in (a). With the choice $k^{2}=\frac{1}{2}(N+\delta)$, i.e.,

$$
k^{2}=\frac{1}{2}\left(n \phi^{2}+n \phi^{2}\left(\frac{1}{\cosh \phi}\right)^{n-1}\right)
$$

each member of the sequence (20) satisfies $1 / \cosh \phi<u^{m}(x)<1$. In order to show this, we can use induction. First, observe that $1 / \cosh \phi<u^{0}(x)<1$. Next, assume that, for some $r>0, u^{r}(x)$ satisfies $1 / \cosh \phi<u^{r}(x)<1$ for all $x \in[0,1]$. We then have, from (20b),

$$
\begin{aligned}
u^{r+1}(x) & \leqslant \frac{\cosh k x}{\cosh k}+\int_{0}^{1} g_{k}(x, \xi) \cdot k^{2} d \xi \\
& =\frac{\cosh k x}{\cosh k}+k^{2}\left(\frac{\cosh k-\cosh k x}{k^{2} \cosh k}\right)=1 .
\end{aligned}
$$

With $k^{2}$ given by (23), we also have

$$
k^{2} u^{r}(x)-\phi^{2}\left[u^{r}(x)\right]^{n}>k^{2} u^{r}(x)-\phi^{2}\left[u^{r}(x)\right]^{2}
$$

*** For the present purpose we could also choose $u^{0}(x)=1 / \cosh \phi$. 
for all $n \geqslant 2$. Furthermore,

$$
k^{2} u^{r}(x)-\phi^{2}\left[u^{r}(x)\right]^{2} \geqslant k^{2}(1 / \cosh \phi)-\phi^{2}(1 / \cosh \phi)^{2}
$$

for all $x \in[0,1]$. To see this consider the parabola $s(z)=k^{2} z-\phi^{2} z^{2}$ in the interval $1 / \cosh \phi \leqslant z \leqslant 1$. The inequality above is true if the minimum value of the parabola in the interval $1 / \cosh \phi \leqslant z \leqslant 1$ occurs at the left-hand end $z=$ $1 / \cosh \phi$. Note that the zeros are at $z=0$ and $z=k^{2} / \phi^{2}$, and the maximum occurs at $z=k^{2} /\left(2 \phi^{2}\right)$. From (23), $k^{2} / \phi^{2}>1, n \geqslant 2$, and therefore $k^{2} /\left(2 \phi^{2}\right)>$ $1 / 2$. The minimum value is at $1 / \cosh \phi$ if $k^{2} / \phi^{2}-1>1 / \cosh \phi$ or, in other words, if

$$
\frac{n}{2}\left(1+\left(\frac{1}{\cosh \phi}\right)^{n-1}\right)-1>\frac{1}{\cosh \phi},
$$

which can be rearranged as

$$
\frac{n}{2} \frac{\left(1+(1 / \cosh \phi)^{n-1}\right)}{(1+(1 / \cosh \phi))}>1
$$

Note that for $n=2$ equality holds. Also the left-hand side is always greater than $n\left(\frac{1}{2}\right) / 2$ which is greater than or equal to one if $n>4$. By applying elementary calculus, one can also show that when $n=3$ the left-hand side has a minimum in the interval $0<1 / \cosh \phi \leqslant 1$ which occurs when $1 / \cosh \phi=\sqrt{2}-1$. The value of that minimum is greater than one. Therefore the minimum value of the parabola $k^{2} z-\phi^{2} z^{2}$ in the interval $1 / \cosh \phi \leqslant z \leqslant 1$ occurs at $z=1 / \cosh \phi$ for $n=$ $2,3, \ldots$ Finally, if $\phi^{2} \neq 0$ and $n \geqslant 2$, then $k^{2}>\phi^{2}$ and

$$
k^{2} u^{r}(x)-\phi^{2}\left[u^{r}(x)\right]^{n} \geqslant k^{2}(1 / \cosh \phi)-\phi^{2}(1 / \cosh \phi)^{2}>0 .
$$

Thus, from (20b),

$$
\begin{aligned}
u^{r+1}(x) & \geqslant \frac{\cosh k x}{\cosh k}+\int_{0}^{1} g_{k}(x, \xi)\left[k^{2}(1 / \cosh \phi)-\phi^{2}(1 / \cosh \phi)^{2}\right] d \xi \\
& =\frac{\cosh k x}{\cosh k}+\left(\frac{k^{2}}{\cosh \phi}-\frac{\phi^{2}}{(\cosh \phi)^{2}}\right) \cdot\left(\frac{\cosh k-\cosh k x}{k^{2} \cosh k}\right) \\
& =\frac{\cosh k x}{\cosh k}\left(1-\frac{1}{\cosh \phi}\right)+\frac{1}{\cosh \phi}-\frac{\phi^{2}}{k^{2}(\cosh \phi)^{2}}\left(1-\frac{\cosh k x}{\cosh k}\right) \\
& \geqslant \frac{1}{\cosh \phi}+\frac{1}{\cosh k}\left(1-\frac{1}{\cosh \phi}\right)-\frac{\phi^{2}}{k^{2}(\cosh \phi)^{2}}\left(1-\frac{1}{\cosh k}\right) \\
& \geqslant 1 / \cosh \phi
\end{aligned}
$$

if

$$
\frac{\cosh \phi-1}{\cosh k-1} \geqslant \frac{\phi^{2}}{k^{2} \cosh \phi} .
$$

This last inequality inay not be satisfied for all values of $\phi$. We shall restrict the problem to values of $\phi$ that satisfy the inequality (26). Then, by induction, each member of the sequence (20) satisfies $1 / \cosh \phi<u^{m}(x)<1$ for all $x \in[0,1]$.

The conditions in (a) and (b) above are not exactly the same as those in Theorem

2. However, when (c) is satisfied, we can use arguments similar to the arguments in 
the proofs of Theorem 1, Theorem 2, and Keller's theorem to conclude that a unique solution of (16) for $n \geqslant 2$ exists, satisfying $1 / \cosh \phi<u(x)<1$ for all $x \in[0,1]$. It is given by the limit of the sequence (20). In fact, the difference $e^{m+1}(x)$ for the sequence (20) will have the same form as (7), and, in particular with $k^{2}$ given by $(23)$ and $g_{k}(x, \xi)$ by $(19 b)$,

$$
\begin{aligned}
\left|e^{m+1}(x)\right| & \leqslant \int_{0}^{1} g_{k}(x, \xi)\left[\frac{1}{2}\left(n \phi^{2}-n \phi^{2}(1 / \cosh \phi)^{n-1}\right)\right] d \xi\left\|e^{m}\right\| \\
& =\frac{n}{2} \phi^{2}\left(1-(1 / \cosh \phi)^{n-1}\right) \cdot\left\{\frac{\cosh k-\cosh k x}{k^{2} \cosh k}\right\}\left\|e^{m}\right\| \\
& \leqslant \bar{\mu}_{k}\left\|e^{m}\right\|, \quad m=1,2, \ldots,
\end{aligned}
$$

where

$$
\bar{\mu}_{k}=\left\{\frac{1-(1 / \cosh \phi)^{n-1}}{1+(1 / \cosh \phi)^{n-1}}\right\}\left(1-\frac{1}{\cosh k}\right)
$$

for all $x \in[0,1]$.

For comparison, observe that the function $f(x, u)$ for problem (16) will satisfy Keller's condition on $\partial f / \partial u$ with $N=n \phi^{2}$ for all $x \in[0,1]$, and all $u$ such that $1 / \cosh \phi \leqslant u(x) \leqslant 1$ in $[0,1]$. Furthermore, if we had used

$$
k^{2}=n \phi^{2}
$$

in (c) above (the optimal choice indicated by Keller's conditions) then we would have

$$
\left|e^{m+1}(x)\right| \leqslant \mu_{k}\left\|e^{m}\right\|, \quad m=1,2, \ldots,
$$

where

$$
\mu_{k}=\left(1-\frac{1}{\cosh k}\right)
$$

for all $x \in[0,1]$.

Note the difference in the contractive parameters $\mu_{k}$ and $\bar{\mu}_{k}$ in the following table.

TABLE 1

\begin{tabular}{|ccccccc|}
\hline$\phi^{2}$ & $\frac{1}{\cosh \phi}$ & $n$ & $\begin{array}{c}k^{2} \\
\text { Eq. }(29)\end{array}$ & $\mu_{k}$ & $\begin{array}{c}k^{2} \\
\text { Eq. }(23)\end{array}$ & $\bar{\mu}_{k}$ \\
\hline 2.25 & .42 & 2 & 4.50 & .76 & 3.20 & .28 \\
2.25 & .42 & 3 & 6.75 & .85 & 3.97 & .51 \\
2.25 & .42 & 4 & 9.00 & .90 & 4.83 & .67 \\
6.25 & .16 & 2 & 12.50 & .94 & 7.25 & .63 \\
6.25 & .16 & 3 & 18.75 & .97 & 9.62 & .86 \\
6.25 & .16 & 4 & 25.00 & .99 & 12.55 & .93 \\
\hline
\end{tabular}

To obtain a numerical solution of the limit of the sequence (20), $u(x)$ can be approximated by the discrete solution $\tilde{u}_{0}=u(0), \tilde{u}_{1}=u\left(x_{1}\right), \ldots, \tilde{u}_{J-1}=u\left(x_{J-1}\right)$, $\tilde{u}_{J}=u(1)$ on a uniform grid, $h=1 / J, x_{1}=h, x_{2}=2 h, \ldots, x_{J-1}=(J-1) h$, $x_{J}=1$, where $\tilde{u}_{i}$ is the solution to

$$
\tilde{u}_{i}=\frac{\cosh k x_{i}}{\cosh k}+\sum_{j=0}^{J} \alpha_{j} g_{k}\left(x_{i}, x_{j}\right)\left[k^{2} \tilde{u}_{j}-\phi^{2}\left(\tilde{u}_{j}\right)^{n}\right], \quad i=0,1, \ldots, J,
$$


and $\alpha_{0}=\alpha_{J}=h / 2, \alpha_{j}=h, j=1, \ldots, J-1$. Of course, this is an evaluation of the integral by the trapezoidal rule. According to Keller [3] this should yield accuracy on the order of $h^{2}$ where $h=1 / J$. To compute the approximation, a sequence of net functions $\left\{\tilde{u}_{i}^{m}\right\}, m=0,1, \ldots$ can be defined as

$$
\begin{gathered}
\tilde{u}_{i}^{0}=\cosh \phi x_{i} / \cosh \phi, \quad i=0,1, \ldots, J, \\
\tilde{u}_{i}^{m+1}=\frac{\cosh k x_{i}}{\cosh k}+\sum_{j=0}^{J} \alpha_{j} g_{k}\left(x_{i}, x_{j}\right)\left[k^{2} \tilde{u}_{i}^{m}-\phi^{2}\left[\tilde{u}_{i}^{m}\right]^{n}\right], \\
i=0,1, \ldots, J,
\end{gathered}
$$

where $\alpha_{0}=\alpha_{J}=h / 2$ and $\alpha_{j}=h, j=1, \ldots, J-1$. By arguments similar to those given by Keller [3], one can show that the limit of the above sequence exists and is the unique solution of (31). Also, as $J \rightarrow \infty$, the contractive parameter for the sequence (32) will converge to $\bar{\mu}_{k}$ with $k$ defined by (23) (or $\mu_{k}$ with $k$ defined by (29)).

If we assume that $J$ is sufficiently large that the contractive parameter for the sequence (32) is approximately the same as the one for (20), then the difference between the effects of $\bar{\mu}_{k}$ and $\mu_{k}$ can be seen in the following comparison. Consider the case $\phi^{2}=2.25$ and $n=2$. If $\left\|e^{1}\right\|<1$, then the relation $\left\|e^{m+1}\right\|<\left(\mu_{k}\right)^{m}\left\|e^{1}\right\|$ implies that it could take as many as 34 iterations to get the difference in successive iterates on the order of $10^{-4}$. However, the relation $\left\|e^{m+1}\right\|<\left(\bar{\mu}_{k}\right)^{m}\left\|e^{1}\right\|$ implies that it would take at most 8 iterations. A more significant difference would be in a case where $\phi^{2}=6.25$ and $n=2$. In this case, with $\left\|e^{1}\right\|<1$, as many as 148 iterations may be necessary to have $\left\|e^{m+1}\right\|<10^{-4}\left\|e^{1}\right\|$ with $k$ defined by (29). With the value of $k$ given by (23), it would only take at most 20. For graphical results, see [2].

Acknowledgement. The author would like to thank the referee for his criticism and suggestions.

\footnotetext{
Department of Mathematical Sciences

Academic Division

Virginia Commonwealth University

Richmond, Virginia 23284
}

1. R. ARIS, The Mathematical Theory of Diffusion and Reaction in Permeable Catalysis, Vol. I, Clarendon Press, Oxford, London, 1975, pp. 101-239.

2. J. A. De Simone \& J. A. Pennline, “A new asymptotic analysis of the $n$th order reaction-diffusion problem: Analytical and numerical studies," Math. Biosci., v. 40, 1978, pp. 303-318.

3. H. B Keller, Numerical Methods for Two-Point Boundary Value Problems, Blaisdell, Waltham, Mass., 1968, pp. 106-127. 